UNIVERSIDADE NILTON LINS

CURSO DE GRADUAÇÃO EM FISIOTERAPIA

KAREN KAROLINE FERNANDES MOTA

VENTILAÇÃO NÃO INVASIVA EM PACIENTES COM DOENÇA PULMONAR OBSTRUTIVA CRÔNICA (DPOC) NO AMBIENTE HOSPITALAR:

REVISÂO DE LITERATURA 
KAREN KAROLINE FERNANDES MOTA

VENTILAÇÃO NÃO INVASIVA EM PACIENTES COM DOENÇA PULMONAR OBSTTRUTIVA CRÔNICA (DPOC) NO AMBIENTE HOSPITALAR:

REVISÂO DE LITERATURA

Projeto de pesquisa apresentado à disciplina de Trabalho de Conclusão de Curso II, na exigência para obtenção do grau de Bacharel em Fisioterapia.

Orientadora: Prof. Esp. Pricila Cordeiro. 
KAREN KAROLINE FERNANDES MOTA

\section{VENTILAÇÃO NÃO INVASIVA EM PACIENTES COM DOENÇA PULMONAR OBSTTRUTIVA CRÔNICA (DPOC) NO AMBIENTE HOSPITALAR: \\ REVISÂO DE LITERATURA}

Trabalho de Conclusão de Curso, orientado pela Prof. Esp. Pricila Cordeiro, apresentado como requisito para obtenção do grau de Bacharel em Fisioterapia, pela Universidade Nilton Lins.

Aprovado em __ de ___ de 2020

BANCA EXAMINADORA

Prof. Esp. PRICILA CORDEIRO

Orientadora

Professor Convidado 1

Professor Convidado 2 
M917v Mota, Karen Karoline Fernandes

VENTILAÇÃo NÃo INVASIVA EM PACIENTES COM DOENÇA PULMONAR OBSTRUTIVA CRÔNICA (DPOC) NO AMBIENTE HOSPITALAR: REVISÃO DE LITERATURA / Karen Karoline Fernandes Mota. - Manaus: Universidade Nilton Lins - UNL, 2020.

$35 f$.

Trabalho de Conclusäo de Curso (EISIOTERAPIA) Universidade Nilton Ling - UNL: Manaus, 2020.

Orientador (a): Esp. Pricila Cordeiro

1. Palavras-chave: a. Ventilaçäo Nảo Invasiva; b. Dispneia; C. DPOC. I. Titulo.

$\operatorname{CDU} 615.8$

Gerada automaticamente pelo modulo ficha.online

mediante dados fornecidos pelo(a) autor(a). 


\section{RESUMO}

Introdução: A doença pulmonar obstrutiva crônica (DPOC) é caracterizada pela presença de obstrução ou limitação crônica do fluxo aéreo que não é totalmente reversível. O uso da ventilação não invasiva (VNI) tem sido eficaz no tratamento de pacientes com DPOC. Objetivo: Analisar os benefícios da Ventilação não invasiva em pacientes com Doença Pulmonar Obstrutiva crônica no ambiente hospitalar. Métodos: Estudo realizado de natureza básica, com abordagem de pesquisa exploratória e característica bibliográfica, para análise de evidências de revisões. Para obtenção destes elementos foram utilizadas as bases de dados: PEDro, LILACS, SciELO e PubMed, tendo como descritores: Ventilação não invasiva, VNI, Doença Pulmonar Obstrutiva Crônica, DPOC. Resultados: A VNI é uma terapêutica que se mostrou eficiente, melhora significantemente a dispneia, reduz a fadiga, melhora os sinais vitais e a troca gasosa, evita a intubação endotraqueal, reduz mortalidade e diminui os dias de hospitalização. Conclusão: Para que o sucesso da VNI em paciente com DPOC no ambiente hospitalar seja efetivado é necessário seguir critérios rigorosos quanto a indicação e contraindicação, a seleção do paciente, seu modo de uso quanto a relação paciente-ventilador-interface de forma sincronizada para melhores resultados, com o intuito de promover melhora na qualidade de vida do paciente.

Palavras-chave: Ventilação não invasiva; Dispneia; DPOC. 


\begin{abstract}
Introduction: Chronic obstructive pulmonary disease (COPD) is characterized by the presence of obstruction or chronic airflow limitation that is not fully reversible. The use of noninvasive ventilation (NIV) has been effective in the treatment of patients with COPD. Objective: To analyze the benefits of noninvasive ventilation in patients with chronic obstructive pulmonary disease in the hospital environment. Methods: A basic study, with an exploratory research approach and bibliographic characteristic, for analysis of evidence of reviews. To obtain these elements, the following databases were used: PEDro, LILACS, SciELO and PubMed, with the following descriptors: Noninvasive ventilation, NIV, Chronic Obstructive Pulmonary Disease, COPD. Results: NIV is a therapy that has been shown to be efficient, significantly improves dyspnea, reduces fatigue, improves vital signs and gas exchange, prevents endotracheal intubation, reduces mortality and decreases hospitalization days. Conclusion: In order for the success of NIV in patients with COPD in the hospital environment to be effective, it is necessary to follow strict criteria regarding indication and contraindication, patient selection, mode of use as to the patient-ventilatorinterface relationship in a synchronized way for better results, in order to promote improvement in the patient's quality of life.
\end{abstract}

Keywords: Noninvasive ventilation; Dyspnea; COPD. 


\section{LISTA DE ILUSTRAÇÕES}

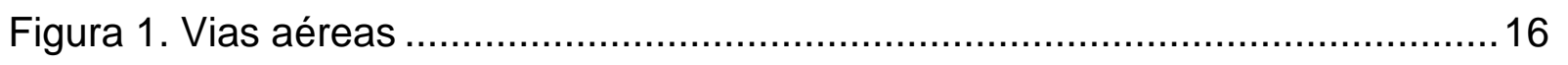




\section{LISTA DE QUADROS}

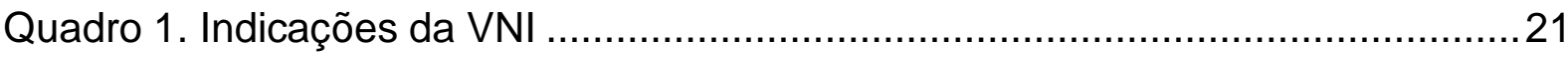

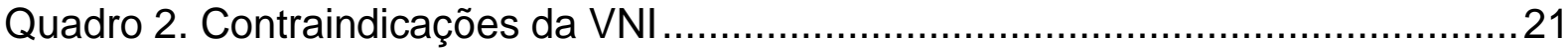




\section{LISTA DE TABELAS}

Tabela 1. Resultados .24 


\section{LISTA DE ABREVIATURAS E SIGLAS}

AVD's - Atividade de vida diárias

BILEVEL - Pressão positiva intermitente

CPAP - Pressão positiva contínua na via aérea

CRF - Capacidade residual funcional

DCV - Doenças cardiovasculares

DPOC - Doença Pulmonar Obstrutiva Crónica

EAP - Edema agudo de pulmão

FiO2 - Fração inspiratória de oxigénio

FR - Frequência respiratória

GOLD - Global iniciative for chronic obstructive lung disease

IMC - Índice de massa corporal

LILACS - Literatura Latino-americana e do Caribe em Ciências da Saúde

$\mathrm{mmHg}$ - Milímetro de mercúrio

NR - Norma Regulamentadora

PaCO2 - Pressão parcial arterial de dióxido de carbono

$\mathrm{PaO} 2$ - Pressão parcial arterial de oxigénio

PAV - Pneumonia Associada a VM

PEDro - Physiotherapy Evidence Database

PEEP - Pressão positiva no final da expiração

$\mathrm{PH}$ - Potencial hidrogeniônico

PubMed - National library of medicine

QDV - Qualidade de vida

SciELO - Scientific Electronic Library Online

SpO2 - Saturação Periférica

TOT - Tubo Orotraqueal

TQT - Traqueostomia

UTI - Unidade de terapia intensiva

VM - Ventilação Mecânica

VMI - Ventilação Mecânica Invasiva

VNI - Ventilação não invasiva

WHODAS - World Health Organization Disabilty Assessment Schedule 


\section{SUMÁRIO}

1 INTRODUÇÃO

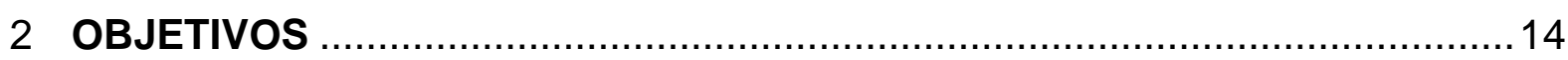

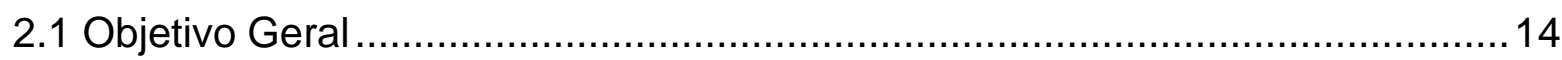

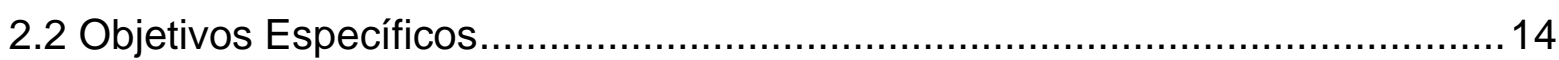

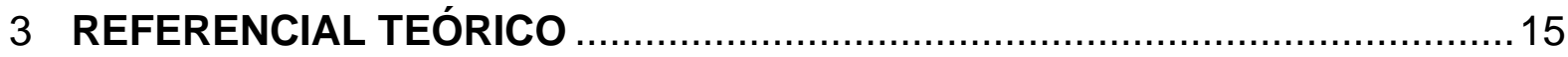

3.1 Anatomofisiologia e Fisiopatologia do Sistema Respiratório …......................15

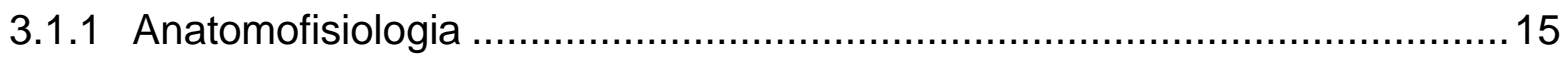

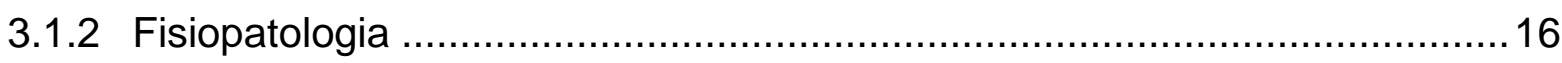

3.2 Doença Pulmonar Obstrutiva Crônica (DPOC) …………............................17

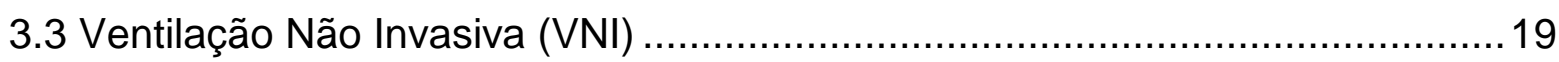

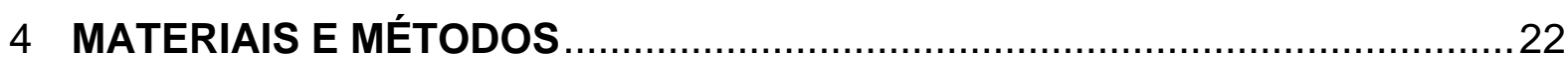

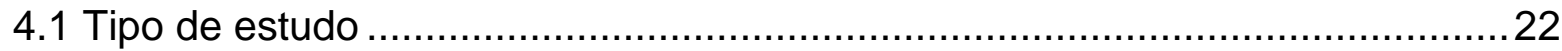

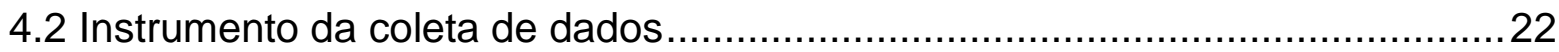

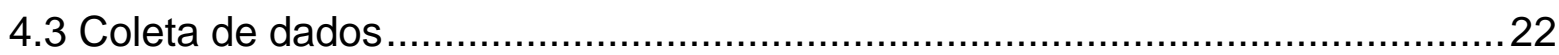

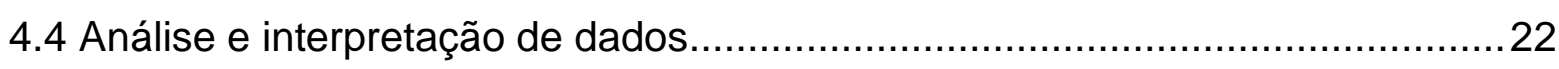

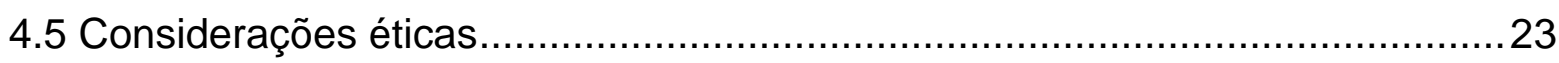

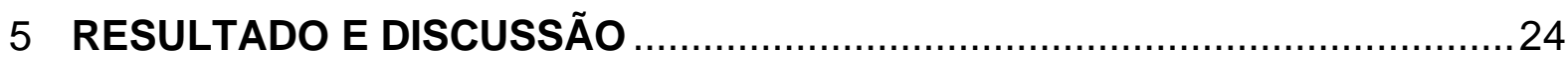

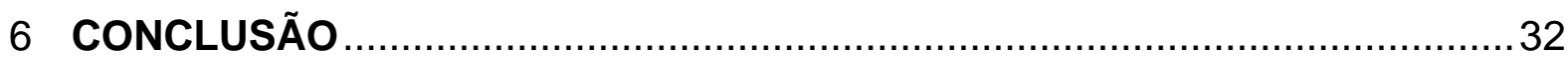

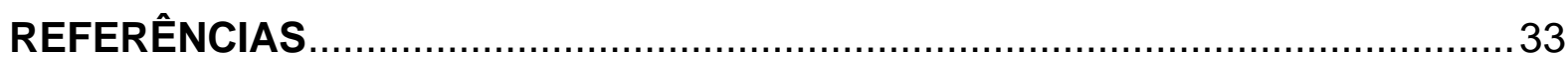




\section{INTRODUÇÃO}

A doença pulmonar obstrutiva crônica (DPOC) é caracterizada pela presença de obstrução ou limitação crônica do fluxo aéreo que não é totalmente reversível. A limitação ao fluxo aéreo é usualmente progressiva e está associada a uma resposta inflamatória anormal dos pulmões a partículas ou gases nocivos (IKE, 2017).

Além da inflamação crônica da via aérea, existem células inflamatórias ativas e o aumento dos níveis plasmáticos de citocinas pró-inflamatórias na circulação sistêmica que, juntamente com o estresse oxidativo, contribuem para as alterações nutricionais e disfunção musculoesquelética (BRAGA, 2017).

Embora a DPOC acometa os pulmões, há diversas manifestações sistêmicas relacionadas a esta enfermidade, que incluem intolerância ao exercício físico, disfunção muscular periférica, alterações nutricionais e exacerbações recorrentes levando a hospitalizações (IKE, 2017).

A doença pulmonar obstrutiva crônica (DPOC) é um dos problemas crônicos de saúde mais comuns. Segundo a Global Initiative for Chronic Obstructive Lung Disease (GOLD), a DPOC é considerada uma doença passível de prevenção e tratamento (VIANA, 2017).

A doença pulmonar obstrutiva crônica está em ascensão no mundo contemporâneo. É uma doença evitável e raramente atribuída à deficiência genética. Vários fatores são responsáveis por este aumento de incidência, como o tabagismo ativo e passivo, a poluição ambiental e ocupacional e a demora de implementação de políticas públicas eficazes para a sua prevenção (AZAMBUJA, 2013).

A prevalência tem grande variação no mundo- de $0,2 \%$ a $37 \%$-, e isto se deve, em parte, às características culturais dos países que emitem relatórios epidemiológicos, dos métodos de diagnóstico estabelecidos no diagnóstico e pela classificação da DPOC que foi utilizada. A prevalência e incidência é maior em homens, especialmente nos idosos acima de 75 anos de idade (AZAMBUJA, 2013).

Nos pacientes com DPOC, os déficits fisiológicos impostos pela natureza progressiva da doença, incluindo limitação ao fluxo aéreo e hiperinsuflação, levam a redução da tolerância ao exercício, que, por sua vez, levam a uma limitação das atividades e ao descondicionamento. Pacientes com DPOC apresentam uma importante fraqueza dos músculos inspiratórios, que pode contribuir para a dispneia e redução do desempenho ao exercício (BRAGA, 2017). 
A progressão da Doença Pulmonar Obstrutiva Crônica (DPOC) está frequentemente associada ao aumento da dispneia e intolerância ao exercício, resultando em impacto negativo na qualidade de vida. A reabilitação pulmonar é uma forma eficiente de minimizar os sintomas causados pela DPOC. A ventilação mecânica não-invasiva (VNI) tem sido usada com o objetivo de reduzir o trabalho dos músculos respiratórios e promover melhores níveis de tolerância ao esforço (ARAÚJO, 2017).

VNI se caracteriza pela oferta de pressão positiva intermitente (Bilevel) ou contínua (CPAP) na via aérea dos pacientes por meio de dispositivo não invasivo (sem que haja colocação de um tubo endotraqueal), normalmente com a utilização de uma interface (máscara) acoplada à boca, nariz ou ambos (MAGALHÃES, 2019).

Em contraste com a Ventilação Mecânica Invasiva (VMI), que se faz por meio de um Tubo Orotraqueal (TOT) ou via Traqueostomia (TQT), a VNI reduz o esforço respiratório e melhora as trocas gasosas, ao mesmo tempo em que mantem a capacidade do paciente de tossir, engolir e conversar (MAGALHÃES, 2019).

O fisioterapeuta exerce um papel relevante no atendimento multidisciplinar oferecido aos pacientes em uma Unidade de Terapia Intensiva (UTI), em ambulatórios e outros serviços. Conforme aponta o III Consenso Brasileiro de Ventilação, de 2017, a atuação desse profissional consiste no atendimento aos pacientes críticos que necessitam de um suporte ventilatório, assim como também pacientes graves que não precisam desse suporte. $O$ intuito é evitar complicações respiratórias e motoras (MAGALHÃES, 2018).

A maioria dos estudos com ventilação com pressão positiva não-invasiva está relacionada a pacientes portadores de doença pulmonar obstrutiva crônica, edema agudo de pulmão, doença torácica restritiva, doença neuromuscular ou apneia do sono; principalmente demonstrando seus benefícios em evitar intubação orotraqueal e ventilação mecânica invasiva, assim como as complicações a ela relacionadas (MAGALHÃES, 2018).

Atualmente com o crescimento da população, fica cada vez mais comum os casos de Doença Pulmonar Obstrutiva Crônica (DPOC). Através dessa pesquisa realizada sobre a Ventilação não invasiva em pacientes com DPOC no ambiente hospitalar, percebe-se a alta incidência desta patologia e o quanto ela interfere na vida diária desses indivíduos. 
O interesse pelo presente estudo é revisar artigos sobre o tratamento da DPOC através da VNI no ambiente hospitalar comprovando sua eficácia, funcionalidade e melhora na qualidade de vida do paciente, a fim de identificar os benefícios, já que a DPOC se trata de uma patologia que se caracteriza pela obstrução do fluxo aéreo, geralmente progressiva e está associada a uma resposta inflamatória anormal dos pulmões à inalação de partículas ou gases tóxicos, causada principalmente pelo tabagismo.

O resultado deste estudo possibilitará melhor planejamento do processo de avaliação e tratamento fisioterapêutico para a utilização da ventilação não invasiva em pacientes com DPOC no ambiente hospitalar.

A revisão gera algumas dúvidas que norteiam o assunto, como: Qual a eficácia da Ventilação não invasiva (VNI) em pacientes com Doença Pulmonar Obstrutiva crônica (DPOC) no ambiente hospitalar? Quais as indicações e contraindicações para a utilização da Ventilação não invasiva (VNI) nesses pacientes? Quais os benefícios da Ventilação não invasiva (VNI) nos pacientes com Doença Pulmonar Obstrutiva crônica (DPOC) no ambiente hospitalar? 


\section{OBJETIVOS}

\subsection{Objetivo geral}

- Analisar os benefícios da Ventilação não invasiva em pacientes com Doença Pulmonar Obstrutiva crônica (DPOC) no ambiente hospitalar.

\subsection{Objetivos específicos}

- Identificar os efeitos da Ventilação não invasiva (VNI);

- Descrever a melhor condição respiratória no paciente com DPOC;

- Apontar o índice de mortalidade em comparação ao uso de Ventilação invasiva no tratamento da exacerbação do DPOC. 


\section{REFERENCIAL TEÓRICO}

\subsection{Anatomofisiologia e Fisiopatologia do Sistema Respiratório}

\subsubsection{Anatomofisiologia}

O sistema respiratório está dividido em vias respiratórias superiores e inferiores. As vias respiratórias superiores incluem os seguintes segmentos: Cavidade nasal, Faringe. As vias respiratórias inferiores são compostas pelas seguintes porções: Laringe, Traqueia, Pulmões. O pulmão direito possui três lobos, enquanto o esquerdo possui apenas dois (PAULSEN, vol. 2, p. 28, 2015).

As cavidades nasais, dois canais ocos separados por uma estrutura cartilaginosa chamada septo nasal, onde é filtrado, umidificado e aquecido. Após passar por esta estrutura é encaminhado para a faringe, um canal que se comunica com o Sistema Digestório e que o conduz à laringe, um tubo muscular formado por cartilagens e que abriga as cordas vocais, músculo responsáveis por produzir o som da voz mediante sua vibração (ULLIO, 2014).

Em seguida é levado para traqueia, outro tubo muscular formado por estruturas cartilaginosas que se bifurcam formando os brônquios. Chegando aos brônquios é conduzido por ramificações chamadas bronquíolos atingindo pequenos sacos de espessura fina, chamadas de alvéolos, onde finalmente é absorvido. Algumas destas estruturas comentadas anteriormente como brônquios, bronquíolos e alvéolos são protegidas por sacos róseos e esponjosos chamado de pulmões (ULLIO, 2014).

O pulmão direito possui três lobos (lobos superior, médio e inferior), separados pela fissura oblíqua e pela fissura horizontal. Por sua vez, o pulmão esquerdo possui dois lobos (superior e inferior) e apresenta apenas uma fissura oblíqua (PAULSEN, vol. 2, p. 34, 2015).

Conforme 0 ar passa pelo nariz, três funções respiratórias distintas são realizadas pelas cavidades nasais: 1) o ar é aquecido nas extensas superfícies das conchas e septo, com área total de cerca de 160 centímetros quadrados (Figura 1);2) o ar é quase completamente umidificado, até mesmo antes de ultrapassar por completo as cavidades nasais; 3) e o ar é parcialmente filtrado. Essas funções em conjunto são chamadas função de condicionamento do ar das vias aéreas superiores. (HALL, 2017). 
Figura 1. Vias aéreas

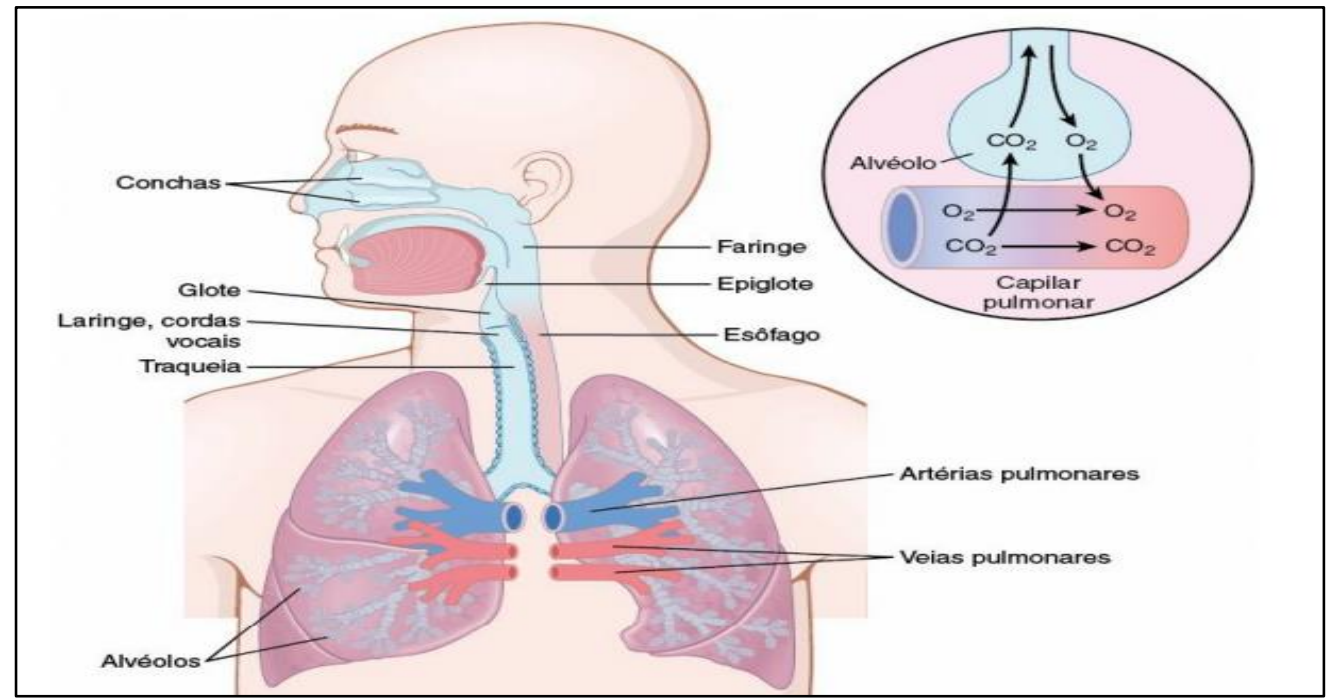

Fonte: HALL, John E. Guyton \& Hall. Tratado de fisiologia médica. 13.ed. Rio de Janeiro: Elsevier Editora, 2017.

\subsubsection{Fisiopatologia}

O termo DPOC compreende um grupo de características clínico-patológicas que têm em comum a obstrução ao fluxo aéreo expiratório, denominadas enfisema pulmonar e bronquite crônica. Diante dessa obstrução pulmonar, os portadores de DPOC sofrem prejuízos na mecânica pulmonar e na musculatura periférica, de modo progressivo, persistente e irreversível. A predominância dessas alterações varia de indivíduo para indivíduo (NOHAMA, 2012).

Quando uma pessoa inspira profundamente logo após uma expiração forçada, o volume de ar no pulmão pode aumentar até quatro vezes. Se a expansão pulmonar fosse uniforme seria necessário um aumento linear das dimensões pulmonares em quase duas vezes (RUFINO, 2013).

Contudo, as diferenças regionais do sistema respiratório dificultam esse resultado matemático. Em parte, isto é explicado pela forma piramidal dos pulmões e pela pressão gravitacional. As maiores expansões ocorrem nos ductos alveolares e nos alvéolos, que mantêm os ângulos das divisões brônquicas estáveis. Isto é denominado de expansão isotrópica (RUFINO, 2013).

A obstrução pulmonar do fluxo expiratório é causada por diversos fatores fisiopatológicos que levam à hiperinsulflação pulmonar. Tais processos tendem a gerar uma diminuição da capacidade inspiratória, devido à dispneia que inicialmente surge durante a atividade física e, com a progressão da doença, também no repouso. Esse fato, além de promover alterações de volumes e da capacidade pulmonar, interfere na atuação, mobilidade e conformidade do diafragma (NOHAMA, 2012). 


\subsection{Doença Pulmonar Obstrutiva Crônica (DPOC)}

A DPOC é definida como uma doença caracterizada por limitação ao fluxo de ar que não é completamente reversível. Inclui as seguintes entidades: 1) o enfisema, uma condição anatomicamente definida caracterizada por destruição e aumento dos alvéolos pulmonares; 2) a bronquite crônica, uma condição definida clinicamente por tosse e expectoração crônicas; 3) a bronquiolite obstrutiva, uma condição em que pequenos bronquíolos estão estreitados (BÁRTHOLO, 2013).

A doença pulmonar obstrutiva crônica (DPOC) gera uma inflamação crônica das vias aéreas centrais e periféricas perante a inalação de gases tóxicos, os pacientes portadores da doença apresentam como característica a redução do fluxo aéreo e diminuição do seu volume expiratório forçado (GOLD, 2015).

A doença pulmonar obstrutiva crônica (DPOC) é uma enfermidade que, embora acometa primariamente os pulmões, traz consequências sistêmicas significativas que levam à intolerância à prática de exercícios físicos e atividades cotidianas. Esse acometimento sistêmico tem sido relacionado à sobrevida e ao estado geral de saúde dos pacientes portadores da doença (DIAS, 2014).

Estudos têm evidenciado o impacto progressivo da DPOC sobre a capacidade respiratória e tolerância ao exercício físico por perda de massa muscular. Consequentemente observa-se redução na qualidade de vida e atividade de vida diária com risco aumentado para a morte, hospitalização e redução da sobrevida (SILVA et al. 2016).

A dispneia gerada pelas alterações no parênquima pulmonar leva os pacientes ao sedentarismo crônico. Esse ciclo de dispneia e sedentarismo promove a hipotrofia muscular periférica e a redução da capacidade aeróbia, resultando em uma demanda ventilatória ainda mais intensa para as atividades dinâmicas, fechando o ciclo denominado dispneia-sedentarismo-dispneia (DIAS, 2014).

A evolução da DPOC caracteriza-se por exacerbações, representado pelo agravamento dos sintomas, principalmente da dispneia, tosse produtiva e expectoração purulenta, episódios que estão diretamente associadas com a mortalidade (GOLD, 2015).

A comorbidade com maior impacto nos portadores da DPOC é a de origem cardiovascular, sendo as manifestações sistêmicas mais encontradas, contribuindo diretamente para a evolução clínica da doença, mortalidade e maiores gastos de 
recursos. Cerca de $37 \%$ dos pacientes com DPOC morrem de coronariopatia ou insuficiência cardíaca, comparado a $34 \%$ de morte apenas pelo acometimento da própria doença. A associação entre doenças cardiovasculares (DCV) e DPOC é geralmente encontrada em DPOC leve a moderado (CHEN et al. 2015).

A prevalência, morbilidade e mortalidade da DPOC variam de acordo com os países e com os diferentes grupos em cada país, mas, geralmente, estão diretamente relacionados com a prevalência do tabagismo, apesar de que, em alguns países, a poluição do ar tem sido também apontada como fator de risco da DPOC (FARIAS, 2015).

Entre as seis principais causas de morte relacionadas ao tabaco, a DPOC é a primeira delas que, apesar de uma queda acentuada nos últimos 25 anos, ainda é responsável por mais óbitos que todas as formas de câncer relacionados ao tabaco juntas (JOSÉ et al., 2017).

A exposição ao tabaco é a principal etiologia da doença, mas outros poluentes ambientais, como partículas e gases (queima de biomassa), também são importantes e são amplificados por fatores que afetam o crescimento pulmonar durante a gestação e a infância. Entretanto, outros fatores também têm sido implicados da gênese da DPOC, uma vez que apenas $15-20 \%$ dos fumantes desenvolvem os sintomas da doença (RABAHI, 2013).

A exposição à biomassa tem sido um desses fatores alvo de inúmeras publicações; em todo o mundo, são quase 3 bilhões de pessoas expostas à fumaça da combustão de biomassa, seja para cozinhar, seja como fonte de energia para o aquecimento doméstico. Nesses locais, incluindo grande parte da população rural do Brasil, a poluição doméstica também é responsável pela etiologia da DPOC (RABAHI, 2013).

Entendendo que a DPOC afeta múltiplos aspetos da vida dos indivíduos, cada vez mais investigadores têm incorporado nos seus estudos medidas de Qualidade de Vida (QDV), como parâmetro adicional na avaliação das intervenções e na decisão por diferentes tratamentos. De fato, a qualidade de vida é hoje um tema de primordial importância, uma vez que viver não é mais e só um problema de subsistência. Trata-se de valorizar e dignificar a vida tomando como aspeto central a saúde ao invés de doença (SILVA, 2011). 


\subsection{Ventilação Não Invasiva (VNI)}

O III Consenso Brasileiro de Ventilação Mecânica define como suporte não invasivo o método de suporte ventilatório que utiliza a pressão positiva sem o uso de tubos traqueais. A ventilação não invasiva (VNI) provê assistência ventilatória com o uso de máscaras na interface paciente-ventilador, isto é, utiliza interface externa. O termo VNI inclui outras formas de assistência ventilatória que evitam qualquer tipo de invasão da via aérea, tal como a ventilação por pressão negativa (CRUZ, 2013).

O suporte ventilatório fornecido pela VM classifica-se em: Ventilação Mecânica Invasiva (VMI) e Ventilação Não-Invasiva (VNI). A primeira oferece suporte ventilatório por meio de tubo oro ou nasotraqueal, enquanto a segunda modalidade utiliza uma máscara como interface entre o paciente e o ventilador artificial (DO NASCIMENTO, 2019).

A VNI tem sido utilizada por reduzir a necessidade de intubação orotraqueal em pacientes com exacerbação da Doença Pulmonar Obstrutiva Crônica (DPOC), Edema Agudo de Pulmão (EAP), pacientes imunossuprimidos e portadores de doenças neuromusculares. A VNI apresenta nível de evidência A para o tratamento de condições clínicas como o EAP e a DPOC (DO NASCIMENTO, 2019).

A VNI é um suporte ventilatório com pressão positiva, no qual a conexão entre o ventilador e o paciente é feita por meio de uma interface. Em pacientes hospitalizados, as interfaces mais utilizadas são os modelos orofacial, nasal ou full face. O sucesso da VNI, além dos critérios de elegibilidade, é dependente da adaptação acurada da interface, do conforto, da aceitação e da otimização do paciente (DE ARAUJO, 2019).

Para Fernández et al., o sucesso de ventilação não-invasiva depende em grande parte do elemento de interface em que a interação do paciente ocorre com o respirador. Um programa flexível, um gatilho ou sensibilidade adequada e um ajuste dos tempos expiratório e inspiratório podem definir o conforto do paciente durante os primeiros minutos de ventilação e é mais fácil convencê-lo a apoiar a máscara (DOS SANTOS, 2017). 
Vale ressaltar que o suporte ventilatório não invasivo cumpre seus objetivos quando assegura uma apropriada sincronia paciente-ventilador, e um fator decisivo para isto é que os respiradores sejam muito sensíveis e que os parâmetros predefinidos correspondam às necessidades de oxigenação e ventilação alveolar. (DOS SANTOS, 2017).

Uma das vantagens da utilização da VNI é a diminuição, ou mesmo a eliminação, da necessidade de intubação, consequentemente diminuindo as complicações associadas a esse método invasivo (trauma nas vias aéreas, infecções nosocomiais, necessidade de sedação) e diminuição dos custos hospitalares (DE ARAUJO, 2019).

A VNI define-se por ser uma técnica ventilatória sem entubação orotraqueal, por pressão positiva, com a finalidade de substituir ou assistir os músculos inspiratórios, e que produz vários efeitos benéficos ao nível do sistema respiratório, tais como: optimização das trocas gasosas, controle da dispneia, repouso da musculatura ventilatória e diminuição do trabalho ventilatório, repercutindo-se na melhoria de qualidade de vida e prognóstico do paciente (CORREIA, 2013).

A VNI tem sido usada no tratamento das doenças obstrutivas crônicas com o objetivo de reduzir o trabalho dos músculos respiratórios, minimizando os efeitos da hiperinsuflação dinâmica e promovendo melhores níveis de tolerância ao esforço. A finalidade primordial do suporte ventilatório artificial é a de interagir com os componentes da caixa torácica de modo a propiciar a sincronia da interface paciente e ventilador, minimizando a dispneia (ARAÚJO, 2017).

A CPAP é o modo mais comum de VNI, tendo sua eficácia comprovada na melhora da dispneia e redução do esforço dos músculos respiratórios. A CPAP aumenta a capacidade residual funcional (CRF) e recruta alvéolos colapsados e pouco ventilados. $O$ aumento na CRF pode também favorecer a complacência pulmonar, diminuindo o trabalho da respiração. Além disso, a CPAP age neutralizando os efeitos da auto PEEP (pressão positiva no final da expiração) de forma a diminuir a compressão dinâmica das vias aéreas na expiração (ARAÚJO, 2017).

A VNI é indicada de acordo com sinais funcionais e clínicos de desconforto respiratório, como resumidamente demonstrado no Quadro 1. Diferentemente das variações encontradas para as indicações e resultados clínicos esperados, existe um consenso geral em relação às contraindicações da VNI - Quadro 2 (CRUZ, 2013). 
Quadro 1. Indicações da VNI

- Deterioração da troca gasosa $(\mathrm{PaO} 2 / \mathrm{FiO} 2<200$ ou SpO2 < 92\%);

- Falência na bomba ventilatória com hipercapnia e acidose respiratória $(\mathrm{PaCO} 2>45 \mathrm{mmHg}$ e $\mathrm{pH}<7,35)$;

- Dispneia com uso da musculatura acessória e/ou respiração paradoxal;

- Taquipneia (frequência respiratória $>24$ incursões por minuto).

Fonte: CRUZ, Mônica R.; ZAMORA, Victor E. C.. Ventilação mecânica não nvasiva.Revista Hospital Universitário Pedro Ernesto, [S.I.], v. 12, n. 3, set. 2013. ISSN 1983-2567. Disponível em:<https://www.e-publicacoes.uerj.br/index.php/revistahupe/article/view/7535/5917>. Acesso em: 14 nov. 2019. doi:https://doi.org/10.12957/rhupe.2013.7535

Quadro 2. Contraindicações da VNI

- Parada cardíaca ou respiratória;

- Arritmia cardíaca instável ou instabilidade hemodinâmica;

- Falência múltipla de órgãos;

- Encefalopatia severa (escala de coma de Glasgow < 10);

- Hemorragia digestiva;

- Cirurgia de face, trauma ou deformidade;

- Pneumotórax não drenado;

- Hipoxemia severa;

- Obstrução de via aérea superior;

- Incapacidade de cooperar e/ou proteger a via aérea;

- Incapacidade de eliminar secreções;

- Risco aumentado de aspiração.

Fonte: CRUZ, Mônica R.; ZAMORA, Victor E. C.. Ventilação mecânica não nvasiva.Revista Hospital Universitário Pedro Ernesto, [S.I.], v. 12, n. 3, set. 2013. ISSN 1983-2567. Disponível em:<https://www.e-publicacoes.uerj.br/index.php/revistahupe/article/view/7535/5917>. Acesso em: 14 nov. 2019. doi:https://doi.org/10.12957/rhupe.2013.7535 


\section{MATERIAIS E MÉTODOS}

\subsection{Tipo de estudo}

O estudo realizado é de natureza básica, com abordagem de pesquisa exploratória e característica bibliográfica, para análise de evidências de revisões sistemáticas sobre o uso da Ventilação Não Invasiva (VNI) no paciente com Doença Pulmonar Obstrutiva Crônica (DPOC) no ambiente hospitalar.

\subsection{Instrumento da coleta de dados}

O trabalho foi coletado através de dados eletronicamente, de acordo com Marconi e Lakatos (2010) é um levantamento geral dos principais trabalhos realizados, através de materiais escritos e eletrônicos, livros, revistas, trabalhos avulsos, artigos científicos, e imprensa escrita, que fornecem dados atualizados e relevantes de acordo com o tema tendo como objetivo que investigador tenha acesso ao material escrito de um determinado assunto, auxiliando na análise e manipulação de sua pesquisa.

\subsection{Coleta de dados}

Os critérios de inclusão desta revisão foi: ensaios randomizados controlados, artigos publicados a partir de 2011 e os critérios de exclusão foi: artigos que tinham sido publicados anteriormente a 2011 e artigos duplicados. Para obtenção destes elementos foram utilizadas as bases de dados: PEDro, LILACS, SciELO e PubMed, tendo como descritores: Ventilação não invasiva, VNI, Doença Pulmonar Obstrutiva Crônica, DPOC.

\subsection{Análise e interpretação de dados}

Para esta etapa a análise dos estudos foi pautada de acordo com o modelo de leitura exploratória proposta por Andrade (2006) cujos principais objetivos analisados estão: a análise textual, em que se realiza um levantamento histórico e uma visão global dos elementos mais relevantes presentes no estudo e a análise interpretativa, que os elementos presentes na pesquisa são analisados de forma crítica de acordo com embasamento científico e os julgamentos filosóficos que surgem em torno 
deste, há um levantamento das discussões dos dados da literatura teórica e empírica.

\subsection{Considerações éticas}

Por se tratar de uma pesquisa de revisão de literatura não foi necessária a submissão do mesmo ao Comitê de Ética em Pesquisas com seres humanos, conforme a resolução n466/12 do Conselho Nacional de Saúde. No entanto houve o comprometimento em citar os autores dos estudos aqui abordados, acatando a determinação da Norma Brasileira Regulamentadora (NR 6023). 


\section{RESULTADO E DISCUSSÃO}

Tabela 1. Resultados

\begin{tabular}{|c|c|c|c|c|c|c|}
\hline Nome Artigo & Ano & Autor & Objetivo geral & Idioma & Metodologia & Conclusão \\
\hline $\begin{array}{l}\text { O uso da ventilação } \\
\text { não-invasiva na } \\
\text { reabilitação } \\
\text { pulmonar } \\
\text { pacientes portadores } \\
\text { da doença pulmonar } \\
\text { obstrutiva crônica. }\end{array}$ & 2017 & $\begin{array}{l}\text { ARAÚJO, Raquel } \\
\text { Borges et al. }\end{array}$ & $\begin{array}{ll}\text { Estudar os efeitos } & \text { da } \\
\text { ventilação não-invasiva } & \text { na } \\
\text { reabilitação pulmonar em } \\
\text { pacientes portadores da } \\
\text { DPOC grau moderado a } \\
\text { severo. }\end{array}$ & Português & $\begin{array}{l}\text { Revisão de } \\
\text { Literatura }\end{array}$ & $\begin{array}{l}\text { A VNI além de apresentar boa } \\
\text { aceitação por parte dos pacientes } \\
\text { parece melhorar a mecânica } \\
\text { respiratória devido à diminuição da } \\
\text { sensação de dispneia, aumentando os } \\
\text { índices de tolerância ao esforço, além } \\
\text { de oferecer maior conforto ao } \\
\text { paciente, com baixos riscos de } \\
\text { complicações. }\end{array}$ \\
\hline $\begin{array}{l}\text { A influência da } \\
\text { ventilação não- } \\
\text { invasiva na } \\
\text { reabilitação } \\
\text { pulmonar do DPOC. }\end{array}$ & 2017 & $\begin{array}{l}\text { BRAGA, } \\
\text { Machado; } \\
\text { CHAGAS } \\
\text { SANTIAGO, } \\
\text { Mariana; } \quad \text { DOS } \\
\text { SANTOS, Michele } \\
\text { Félix. }\end{array}$ & $\begin{array}{l}\text { Analisar os efeitos da VNI } \\
\text { sobre o DPOC na reabilitação } \\
\text { pulmonar. }\end{array}$ & Português & $\begin{array}{l}\text { Artigo } \\
\text { Revisão }\end{array}$ & $\begin{array}{l}\text { Demonstrou que o uso do Suporte } \\
\text { Ventilatório Não- Invasivo, aplicado } \\
\text { durante o exercício físico aos } \\
\text { pacientes com DPOC, vem como } \\
\text { alternativa para melhorar o } \\
\text { desempenho muscular respiratório } \\
\text { gerando aumento na capacidade } \\
\text { funcional e consequentemente } \\
\text { melhora na qualidade de vida. }\end{array}$ \\
\hline $\begin{array}{lr}\text { Risco } & \text { de } \\
\text { comorbidade } & \\
\text { cardiovascular } & \text { em } \\
\text { pacientes } & \text { com }\end{array}$ & 2015 & CHEN, Wenjia et al. & $\begin{array}{l}\text { Quantificar as magnitudes de } \\
\text { associação entre os tipos de } \\
\text { doenças cardiovasculares, }\end{array}$ & Inglês & $\begin{array}{l}\text { Revisão } \\
\text { sistemática e } \\
\text { meta-análise }\end{array}$ & $\begin{array}{l}\text { Os pacientes com DPOC apesentam } \\
\text { maior probabilidade de serem } \\
\text { diagnosticados com doença } \\
\text { cardiovascular, portanto, é necessário }\end{array}$ \\
\hline
\end{tabular}




\begin{tabular}{|c|c|c|c|c|c|c|}
\hline $\begin{array}{l}\text { doença pulmonar } \\
\text { obstrutiva crônica. }\end{array}$ & & & $\begin{array}{l}\text { principais fatores de risco } \\
\text { cardiovascular e DPOC. }\end{array}$ & & & $\begin{array}{l}\text { o desenvolvimento de estratégias para } \\
\text { rastrear e reduzir os riscos } \\
\text { cardiovasculares associados com a } \\
\text { DPOC. }\end{array}$ \\
\hline $\begin{array}{lr}\text { Ventilação } & \text { não } \\
\text { invasiva } & \text { durante } 0 \\
\text { exercício } & \text { em } \\
\text { pacientes } & \text { com } \\
\text { DPOC. } & \end{array}$ & 2013 & CORREIA, S. & $\begin{array}{l}\text { Verificar se a utilização de VNI } \\
\text { durante o exercício é mais } \\
\text { eficaz que exercício sem VNI, } \\
\text { na dispneia, distância } \\
\text { percorrida, gasometria e } \\
\text { estado de saúde, em } \\
\text { pacientes com DPOC. }\end{array}$ & Português & $\begin{array}{l}\text { Revisão } \\
\text { sistemática } \\
\text { com meta- } \\
\text { análise }\end{array}$ & $\begin{array}{l}\text { Verificou-se a influência positiva da } \\
\text { VNI durante o exercício, quer em } \\
\text { termos de respostas agudas ou } \\
\text { crónicas ao exercício, em pacientes } \\
\text { hipercapnicos e normocapnicos. } \\
\text { independentemente da realização de } \\
\text { programas de treino de exercício. }\end{array}$ \\
\hline $\begin{array}{l}\text { Ventilação mecânica } \\
\text { não invasiva. }\end{array}$ & 2013 & $\begin{array}{l}\text { CRUZ, Mônica R.; } \\
\text { ZAMORA, Victor E. } \\
\text { C.. }\end{array}$ & $\begin{array}{l}\text { Revisar a literatura mais } \\
\text { recente sobre as indicações } \\
\text { clínicas e o uso da ventilação } \\
\text { não invasiva. }\end{array}$ & Português & $\begin{array}{l}\text { Artigo } \\
\text { Revista }\end{array}$ & $\begin{array}{l}\text { A utilização da VNI tem sido utilizada } \\
\text { como uma alternativa para evitar a } \\
\text { intubação de pacientes críticos. Seu } \\
\text { uso é recomendado na doença } \\
\text { pulmonar obstrutiva crônica, edema } \\
\text { pulmonar cardiogênico e em pacientes } \\
\text { imunossuprimidos. O sucesso da VNI } \\
\text { depende de fatores como a escolha da } \\
\text { interface, modo ventilatório, interação } \\
\text { paciente-ventilador e monitorização } \\
\text { cuidadosa tanto de sinais objetivos } \\
\text { quanto do conforto e tolerância do } \\
\text { paciente. }\end{array}$ \\
\hline
\end{tabular}




\begin{tabular}{|c|c|c|c|c|c|c|}
\hline $\begin{array}{lr}\text { Fatores } & \text { Preditores } \\
\text { para a } & \text { Falha } \\
\text { Ventilação } & \text { não } \\
\text { Invasiva } & \text { em } \\
\text { Pacientes } & \\
\text { Hospitalizados } & \text { com } \\
\text { Câncer. }\end{array}$ & 2019 & $\begin{array}{l}\text { DE } \quad \text { ARAUJO, } \\
\text { Bianca } \\
\text { al. }\end{array}$ & $\begin{array}{l}\text { Analisar os fatores preditores } \\
\text { para falha da VNI em } \\
\text { pacientes oncológicos; } \\
\text { descrever a mortalidade } \\
\text { hospitalar e a sobrevida global } \\
\text { após internação. }\end{array}$ & Português & $\begin{array}{l}\text { Artigo } \\
\text { Revista }\end{array}$ & $\begin{array}{l}\text { Pacientes que apresentaram infecção } \\
\text { pulmonar tiveram maior probabilidade } \\
\text { em falhar na VNI. Foi observada pior } \\
\text { sobrevida global entre aqueles } \\
\text { pacientes que falharam na VNI. }\end{array}$ \\
\hline $\begin{array}{l}\text { Avaliação da } \\
\text { composição corporal, } \\
\text { capacidade funcional } \\
\text { e função pulmonar } \\
\text { em pacientes com } \\
\text { Doença Pulmonar } \\
\text { Obstrutiva Crônica. }\end{array}$ & 2014 & $\begin{array}{l}\text { DIAS, Fernanda } \\
\text { Dultra et al. }\end{array}$ & $\begin{array}{l}\text { Verificar se há relação entre os } \\
\text { diferentes itens da } \\
\text { composição corporal, como a } \\
\text { capacidade funcional e a } \\
\text { gravidade da doença em } \\
\text { pacientes com DPOC }\end{array}$ & Português & $\begin{array}{l}\text { Estudo } \\
\text { caso }\end{array}$ & $\begin{array}{l}\text { A gravidade da obstrução } \\
\text { apresentada pela alteração no } \\
\text { parênquima pulmonar nos pacientes } \\
\text { com DPOC está relacionada com as } \\
\text { alterações na composição corporal e } \\
\text { com a redução da capacidade } \\
\text { funcional. }\end{array}$ \\
\hline \begin{tabular}{lr}
\multicolumn{2}{l}{ Avaliação do uso de } \\
ventilação & não- \\
invasiva & no \\
desmame & da \\
ventilação & mecânica \\
em & pacientes \\
adultos. &
\end{tabular} & 2019 & $\begin{array}{l}\text { DO NASCIMENTO, } \\
\text { Ana Letícia Santos } \\
\text { et al. }\end{array}$ & $\begin{array}{l}\text { Avaliar o uso da VNI no } \\
\text { processo de desmame da VMI } \\
\text { em pacientes adultos. }\end{array}$ & Português & $\begin{array}{l}\text { Artigo } \\
\text { Revisão }\end{array}$ & $\begin{array}{l}\text { A VNI é uma terapêutica que se } \\
\text { mostrou viável em alguns estudos } \\
\text { para reduzir os riscos de reintubação } \\
\text { em pacientes com diversidade } \\
\text { diagnóstica que apresentaram } \\
\text { insuficiência respiratória aguda após a } \\
\text { extubação. }\end{array}$ \\
\hline $\begin{array}{l}\text { Efeitos da ventilação } \\
\text { não invasiva em } \\
\text { pacientes com }\end{array}$ & 2017 & $\begin{array}{l}\text { DOS SANTOS, } \\
\text { Leandro Andrade et } \\
\text { al. }\end{array}$ & $\begin{array}{l}\text { Analisar a produção científica } \\
\text { nacional e internacional } \\
\text { referente ao uso de ventilação } \\
\text { não invasiva em pacientes }\end{array}$ & $\begin{array}{l}\text { Inglês/ } \\
\text { Português }\end{array}$ & $\begin{array}{l}\text { Revisão de } \\
\text { Literatura }\end{array}$ & $\begin{array}{l}\text { Com bases na pesquisa, as principais } \\
\text { indicações clínicas baseadas em } \\
\text { evidências para o uso de VNI no } \\
\text { ambiente de cuidados intensivos são }\end{array}$ \\
\hline
\end{tabular}




\begin{tabular}{|c|c|c|c|c|c|c|}
\hline $\begin{array}{l}\text { insuficiência } \\
\text { respiratória aguda. }\end{array}$ & & & $\begin{array}{l}\text { com insuficiência respiratória } \\
\text { aguda, de modo a conhecer os } \\
\text { seus efeitos e identificar os } \\
\text { fatores associados ao sucesso } \\
\text { e falha deste método. }\end{array}$ & & & $\begin{array}{l}\text { exacerbações de doença pulmonar } \\
\text { obstrutiva crônica e edema pulmonar } \\
\text { cardiogênico agudo. }\end{array}$ \\
\hline $\begin{array}{l}\text { Qualidade de Vida da } \\
\text { Pessoa com Doença } \\
\text { Pulmonar Obstrutiva } \\
\text { Crónica. }\end{array}$ & 2015 & $\begin{array}{l}\text { FARIAS, Gabriela \& } \\
\text { MARTINS, Rosa. }\end{array}$ & $\begin{array}{l}\text { Conhecer a Qualidade de Vida } \\
\text { do doente com DPOC e } \\
\text { fatores que nela intervêm. }\end{array}$ & Português & Artigo & $\begin{array}{l}\text { A limitação das atividades físicas é o } \\
\text { que mais contribui para o maior } \\
\text { prejuízo na QDV, seguida pelos } \\
\text { sintomas da doença e, por fim, o } \\
\text { impacto psicossocial. Por outro lado, } \\
\text { verificámos que um maior número de } \\
\text { internamentos e um estádio mais } \\
\text { avançado da DPOC contribui para } \\
\text { uma menor QDV nestes doentes. }\end{array}$ \\
\hline $\begin{array}{lr}\text { Mortalidade } & \text { e } \\
\text { incapacidade } & \text { por } \\
\text { doenças } & \\
\text { relacionadas } & \text { à }\end{array}$ & 2017 & $\begin{array}{l}\text { JOSÉ, Bruno Piassi } \\
\text { de São et al. }\end{array}$ & $\begin{array}{l}\text { Analisar as tendências de } \\
\text { mortalidade por doença } \\
\text { pulmonar obstrutiva crônica } \\
\text { (DPOC) e câncer de pulmão, }\end{array}$ & $\begin{array}{l}\text { Português/ } \\
\text { Inglês }\end{array}$ & Artigo & $\begin{array}{l}\text { Ações nacionais nas últimas décadas } \\
\text { têm tido grande efeito na diminuição } \\
\text { da mortalidade de doenças } \\
\text { relacionadas ao tabaco, mas ainda há }\end{array}$ \\
\hline
\end{tabular}




\begin{tabular}{|c|c|c|c|c|c|c|}
\hline $\begin{array}{l}\text { exposição ao tabaco } \\
\text { no Brasil, } 1990 \text { a } \\
2015 .\end{array}$ & & & $\begin{array}{l}\text { lábios, cavidade oral, faringe e } \\
\text { esôfago, no Brasil, entre } 1990 \\
\text { e } 2015 \text {. }\end{array}$ & & & $\begin{array}{l}\text { grandes desafios, principalmente } \\
\text { quando se trata de mulheres e jovens. }\end{array}$ \\
\hline $\begin{array}{lr}\text { Incapacidade } \\
\text { funcional } & \text { de } \\
\text { pacientes } & \text { com } \\
\text { doença } & \text { pulmonar } \\
\text { obstrutiva } & \text { crônica } \\
\text { através } & \text { da } \\
\text { WHODAS. } & \end{array}$ & 2016 & $\begin{array}{l}\text { SILVA, Cássio } \\
\text { Magalhaes et al. }\end{array}$ & $\begin{array}{l}\text { Avaliar a incapacidade } \\
\text { funcional de pacientes com } \\
\text { doença pulmonar obstrutiva } \\
\text { crônica (DPOC) através do } \\
\text { World Health Organization } \\
\text { Disabilty } \\
\text { Schedule (WHODAS). }\end{array}$ & $\begin{array}{l}\text { Português/ } \\
\text { Inglês }\end{array}$ & Artigo & $\begin{array}{l}\text { Observou-se que os participantes com } \\
\text { DPOC leve a grave apresentaram } \\
\text { dificuldade ligeira para a realização da } \\
\text { atividade de vida diária, com exceção } \\
\text { dos domínios de atividade doméstica } \\
\text { e participação com condição } \\
\text { moderada. Observa-se que o sexo } \\
\text { feminino e os menores de } 60 \text { anos } \\
\text { estão em condição de maior } \\
\text { incapacidade. }\end{array}$ \\
\hline
\end{tabular}

Fonte: $\mathrm{O}$ autor. 
De acordo com CHEN, Wenjia et al. (2015) a doença pulmonar obstrutiva crônica (DPOC) é um distúrbio inflamatório sistêmico associado ao aumento da prevalência comórbida de doenças cardiovasculares. Comparado com a população não-DPOC, os pacientes com DPOC apresentaram maior probabilidade de serem diagnosticados com doença cardiovascular incluindo um risco duas a cinco vezes maior de doença cardíaca isquêmica, disritmia cardíaca, insuficiência cardíaca, doenças da circulação pulmonar e doenças das artérias.

Para DIAS, Fernanda Dultra et al. (2014) a doença pulmonar obstrutiva crônica (DPOC) está relacionada a um baixo índice de massa corporal (IMC), à redução da capacidade funcional e à redução da densidade óssea, justificando assim a importância de se avaliar todos esses parâmetros nos pacientes portadores da doença.

Segundo GOLD (2015), a doença pulmonar obstrutiva crônica (DPOC) gera uma inflamação crônica das vias aéreas centrais e periféricas. Sua evolução caracteriza-se por exacerbações, representado pelo agravamento dos sintomas, principalmente da dispneia, tosse produtiva e expectoração purulenta.

De acordo com JOSÉ, Bruno Piassi de São et al. (2017) entre as seis principais causas de morte relacionadas ao tabaco, a DPOC é a primeira delas. Para FARIAS, Gabriela \& MARTINS, Rosa (2015), a prevalência, morbilidade e mortalidade da DPOC variam de acordo com os países e com os diferentes grupos em cada país, mas, geralmente, estão diretamente relacionados com a prevalência do tabagismo.

Para SILVA, Cássio Magalhaes et al. (2016) O déficit de troca gasosa e a desvantagem mecânica ocasionada pela degradação pulmonar característica da DPOC provoca redução da força muscular respiratória e do limiar de dispneia, assim como redução da tolerância ao exercício e da qualidade de vida. A reabilitação física visa principalmente 0 controle da hiperinsuflação pulmonar inerente ao aprisionamento de ar e a redução da dispneia. Os benefícios obtidos a partir da Fisioterapia em indivíduos com DPOC promove a melhora da tolerância ao exercício e da capacidade em realizar as atividades de vida diárias (AVD's).

Segundo ARAÚJO, Raquel Borges et al. (2017) a progressão da Doença Pulmonar Obstrutiva Crônica (DPOC) está frequentemente associada ao aumento da dispneia e intolerância ao exercício, resultando em impacto negativo na qualidade de vida. A reabilitação pulmonar é uma forma eficiente de minimizar os sintomas causados pela DPOC. A ventilação não-invasiva (VNI) tem sido usada com o objetivo 
de reduzir o trabalho dos músculos respiratórios e promover melhores níveis de tolerância ao esforço.

De acordo com BRAGA, Danielle Machado; DAS CHAGAS SANTIAGO, Mariana; DOS SANTOS, Michele Félix (2017), a ventilação não-invasiva (VNI) na reabilitação pulmonar melhora significantemente a dispneia, o desempenho ao exercício, reduz a fadiga, melhora os sinais vitais e a troca gasosa, evita a intubação endotraqueal, reduz mortalidade e diminui os dias de hospitalização. Efeitos adversos da reabilitação pulmonar são raros e pouco documentados como: lesões musculoesqueléticas, broncoespasmo induzido pelo exercício e infarto agudo do miocárdio. Porém, a VNI pode oferecer inúmeros benefícios aos pacientes com DPOC sobre o sistema cardiovascular e muscular.

Segundo CORREIA, S (2013), ao longo da última década, a VNI tem sido considerada como um tratamento para pacientes com DPOC e insuficiência respiratória global associada, sendo atualmente uma medida terapêutica primária nos doentes com DPOC e falência respiratória crónica. Em situações agudas, esta terapia provou ser eficaz e é atualmente considerada um tratamento standard, melhorando as trocas gasosas, dispneia, sobrevida, e reduzindo a necessidade de entubação. Um dos principais objetivos da VNI é a diminuição da sobrecarga da musculatura ventilatória e optimização das trocas gasosas.

De acordo com CRUZ, Mônica R.; ZAMORA, Victor E. C (2013), o uso da ventilação não invasiva provê assistência ventilatória sem necessidade de via aérea artificial, com o uso de máscaras na interface paciente-ventilador. Sua indicação é bem estabelecida na doença pulmonar obstrutiva crônica. A escolha correta da interface, do modo ventilatório e a monitorização disponível na unidade de terapia intensiva otimizam o conforto e a interação paciente-ventilador com melhores resultados clínicos.

Segundo DE ARAUJO, Bianca Paraiso et al. (2019) o interesse no uso da VNI no cenário da UTI, enfermarias e emergência aumentou muito nos últimos anos. Desde que não haja contraindicações, a VNI pode ser um tratamento de primeira linha. Uma das vantagens da utilização da VNI é a diminuição, ou mesmo a eliminação, da necessidade de intubação, consequentemente diminuindo as complicações associadas a esse método invasivo (trauma nas vias aéreas, infecções nosocomiais, necessidade de sedação) e diminuição dos custos hospitalares. 
De acordo com DO NASCIMENTO, Ana Letícia Santos et al. (2019) o suporte ventilatório fornecido pela VM classifica-se em: Ventilação Mecânica Invasiva (VMI) e Ventilação Não-Invasiva (VNI). Um tempo prolongado de permanência na VMI pode ocasionar danos aos pacientes, tais como, lesão pulmonar induzida pela VM, Pneumonia Associada a VM (PAV), aumento no tempo de permanência na UTI e aumento nos índices de mortalidade. A VNI é útil por reduzir o período de tempo da VMI em pacientes com recorrentes falhas no processo de desmame, diminui o risco de adquirir infecções nosocomiais e o tempo de permanência na UTI, além de ser utilizada por reduzir a necessidade de intubação orotraqueal em pacientes com exacerbação da Doença Pulmonar Obstrutiva Crônica (DPOC).

Segundo DOS SANTOS, Leandro Andrade et al. (2017) as principais indicações clínicas baseadas em evidências para o uso de VNI no ambiente de cuidados intensivos são exacerbações de doença pulmonar obstrutiva crônica e edema pulmonar cardiogênico agudo. Para Fernández et al., o sucesso de ventilação não-invasiva depende em grande parte do elemento de interface em que a interação do paciente ocorre com o respirador, e um fator decisivo para isto é que os respiradores sejam muito sensíveis e que os parâmetros predefinidos correspondam às necessidades de oxigenação e ventilação alveolar. 


\section{CONCLUSÃo}

O presente estudo mostrou que a ventilação não invasiva (VNI) é um recurso que no tratamento de pacientes com DPOC no ambiente hospitalar tem como finalidade a redução do trabalho dos músculos respiratórios, diminuição da fadiga, diminuição do desconforto causado pela dispneia, redução de custo hospitalar, além de promover o aumento da capacidade funcional.

O índice de mortalidade comparado ao uso da ventilação mecânica invasiva é menor, pois evita possíveis complicações e infecções respiratórias. E nas exacerbações da DPOC, a VNI ainda melhora os sinais vitais e a troca gasosa, podendo prevenir a intubação endotraqueal, reduzir complicações respiratórias, reduzir a mortalidade e a diminuição dos dias de hospitalização.

Contudo, para que o sucesso da VNI em paciente com DPOC no ambiente hospitalar seja efetivado é necessário seguir critérios rigorosos quanto a indicação e contraindicação, a seleção do paciente, seu modo de uso quanto a relação pacienteventilador-interface de forma sincronizada para melhores resultados, com o intuito de promover melhora na qualidade de vida do paciente. 


\section{REFERÊNCIAS}

ARAÚJO, Raquel Borges et al. O uso da ventilação não-invasiva na reabilitação pulmonar em pacientes portadores da doença pulmonar obstrutiva crônica: uma revisão de literatura. Fisioterapia em Movimento, v. 18, n. 1, 2017.

AZAMBUJA, Renato et al. Panorama da doença pulmonar obstrutiva crônica. Revista Hospital Universitário Pedro Ernesto, v. 12, n. 2, 2013.

BÁRTHOLO, Rogério M. Diferenças clínicas entre asma e doença pulmonar obstrutiva crônica. Revista Hospital Universitário Pedro Ernesto, v. 12, n. 2, 2013.

BRAGA, Danielle Machado; DAS CHAGAS SANTIAGO, Mariana; DOS SANTOS, Michele Félix. A influência da ventilação não-invasiva na reabilitação pulmonar do DPOC. Revista Científica do Hospital Central do Exército (HCE), n. 1, p. 5-12, 2017.

CHEN, Wenjia et al. Risk of cardiovascular comorbidity in patients with chronic obstructive pulmonary disease: a systematic review and meta-analysis. The Lancet Respiratory medicine, v. 3, n. 8, p. 631-639, 2015.

CORREIA, S. Ventilação não invasiva durante o exercício em pacientes com DPOC: revisão sistemática com meta-análise. 2013. Tese de Doutorado. Escola Superior de Tecnologia da Saúde de Lisboa.

CRUZ, Mônica R.; ZAMORA, Victor EC. Ventilação mecânica não invasiva. Revista Hospital Universitário Pedro Ernesto, v. 12, n. 3, 2013.

DE ARAUJO, Bianca Paraiso et al. Fatores Preditores para a Falha da Ventilação não Invasiva em Pacientes Hospitalizados com Câncer. Revista Brasileira de Cancerologia, v. 65, n. 1, 2019.

DE OLIVEIRA, Paulo Cesar. Apresentações clínicas da DPOC. Pulmão RJ, v. 22, n. 2, p. 15-18, 2013.

DIAS, Fernanda Dultra et al. Avaliação da composição corporal, capacidade funcional e função pulmonar em pacientes com Doença Pulmonar Obstrutiva Crônica. Fisioterapia e Pesquisa, v. 21, n. 1, p. 10-15, 2014.

DO NASCIMENTO, Ana Letícia Santos et al. Avaliação do uso de ventilação não-invasiva no desmame da ventilação mecânica em pacientes adultos-revisão integrativa. Revista Pesquisa em Fisioterapia, v. 9, n. 1, p. 139-149, 2019.

DOS SANTOS, Leandro Andrade et al. Efeitos da ventilação não invasiva em pacientes com insuficiência respiratória aguda: uma revisão integrativa. Revista Ciência \& Saberes-Facema, v. 3, n. 3, p. 642-648, 2017.

FARIAS, Gabriela; MARTINS, Rosa. Qualidade de vida da pessoa com doença pulmonar obstrutiva crônica. Millenium-Journal of Education, Technologies, and Health, n. 48, p. 195-209, 2016.

GOLD Gifcold. Global Strategy for Diagnosis, Management and Prevention of CODP. São Paulo 2015.

HALL, John E. Guyton \& Hall. Tratado de fisiologia médica. 13. ed. Rio de Janeiro: Elsevier Editora, 2017.

IKE, Daniela et al. Efeitos do exercício resistido de membros superiores na força muscular periférica e na capacidade funcional do paciente com DPOC. Fisioterapia em Movimento, v. 23, n. 3, 2017.

JOSÉ, Bruno Piassi de São et al. Mortalidade e incapacidade por doenças relacionadas à exposição ao tabaco no Brasil, 1990 a 2015. Revista Brasileira de Epidemiologia, v. 20, p. 75-89, 2017.

MAGALHÃES, Fransuele Lopes dos Santos; SOARES, Denise Josino. Os benefícios da Ventilação Não Invasiva nos pacientes internados na UTI e em ambulatórios. 2018. 
MAGALHÃES, Ricardo Siufi et al. Importância do intervalo de tempo" porta-máscara" no uso de ventilação não invasiva nas exacerbações agudas de pacientes com DPOC na unidade de emergência do Hospital de Clínicas da Unicamp. 2019.

NOHAMA, Percy; JORGE, Rafaela Fernanda; VALENGA, Marcelo Henrique. Efeitos da estimulação diafragmática transcutânea sincronizada em pacientes com doença pulmonar obstrutiva crônica (DPOC): um estudo piloto. Rev. Bras. Eng. Bioméd., Rio de Janeiro, v. 28, n. 2, p. 103-115, June 2012.

PAULSEN, F.; WASCHKE, J. Sobotta: atlas de anatomia humana: órgãos internos. Vol. 2. In: Sobotta: atlas de anatomia humana: órgãos internos. 23. ed. Rio de Janeiro: Guanabara Koogan, 2015. p. 28; 34.

RABAHI, Marcelo Fouad. Epidemiologia da DPOC: enfrentando desafios. 2013.

RUFINO, Rogério; DA COSTA, Cláudia Henrique. Patogenia da doença pulmonar obstrutiva crônica. Revista Hospital Universitário Pedro Ernesto, [S.I.], v. 12, n. 2, jun. 2013.

SILVA, CMS et al. Incapacidade funcional de pacientes com doença pulmonar obstrutiva crônica através da WHODAS. Revista Acta Fisiátrica, v. 23, n. 3, p. 125-129, 2016.

SILVA, Maíra Shiramizu da. Qualidade de Vida relacionada à saúde de pacientes com Doença Pulmonar Obstrutiva Crónica. 2011. Tese de Doutorado. Universidade de São Paulo.

ULLIO, P. Construindo e Analisando O Sistema Respiratório. Ciências em Tela, Rio de Janeiro, Volume 7, número 1, 2014.

VIANA, Renata Cristina Teixeira Pinto et al. Exacerbação de doença pulmonar obstrutiva crônica na unidade de terapia intensiva: avaliação clínica, funcional e da qualidade de vida na alta e após 3 meses de seguimento. Rev. bras. ter. intensiva, v. 29, n. 1, p. 47-54, 2017. 